\title{
ANALISIS DE DATOS DE ENSAYOS DE DESCOMPOSICION Y MINERALIZACION DE RESIDUOS DE MALEZAS EN CAFÉ USANDO CANASTAS
}

\section{DATA ANALYSIS FROM DECOMPOSITION AND MINERALIZATION OF WEED BIOMASS EXPERIMENT IN COFFEE USING LITTER BAGS}

\section{Aguilar-Bustamante Víctor}

Victor.Aguilar@una.edu.ni / Universidad Nacional Agraria (UNA)

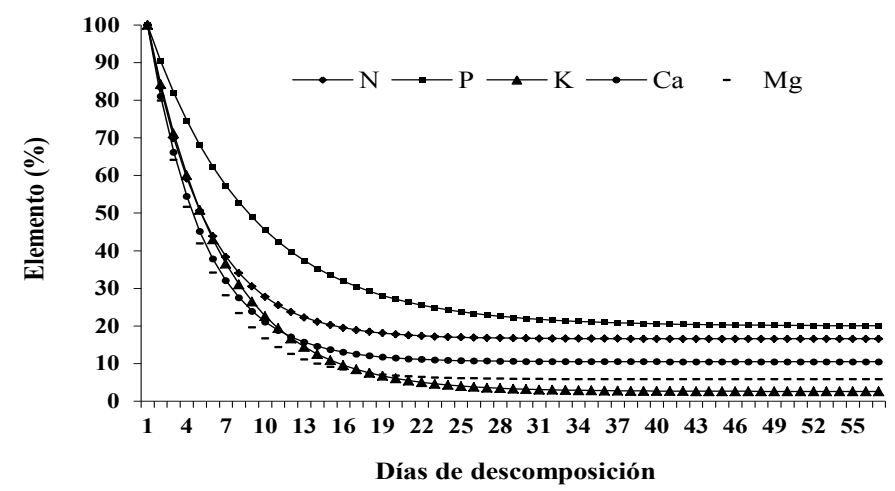

\section{RESUMEN}

Conociendo la descomposición y mineralización de los residuos vegetales en el suelo se puede mejorar el manejo de plantaciones de café que crecen bajo sombra de árboles maderables, de servicios o de uso múltiple. Los beneficios que se puede atribuir a los residuos vegetales cuando son depositados en el suelo, son entre otros los de reducir el impacto de la lluvia evitando así la pérdida de la capa superficial del suelo, mejorar la fertilidad del suelo a través del reciclaje de nutrientes y minimizar el uso de productos químicos como herbicidas al reducir la competencia por espacio y nutrientes entre las malezas y el cultivo de café. El presente estudio fue realizado en la época lluviosa del año 2000 con el objetivo de conocer la velocidad de descomposición y liberación de nutrientes de los diferentes componentes de las principales malezas de los cafetales del pacífico sur de Nicaragua. Canastas de descomposición de $30 \mathrm{~cm}$ de largo, $30 \mathrm{~cm}$ de ancho y $2.5 \mathrm{~cm}$ de alto con malla de orificios de cinco $\mathrm{mm}$ en la parte superficial y un mm en la parte inferior se llenaron con $30 \mathrm{~g}$ de hojas de malezas, $30 \mathrm{~g}$ de tallos de malezas y el tercer tratamiento consistió en la mezcla de $15 \mathrm{~g}$ de hojas y $15 \mathrm{~g}$ de tallos de las mismas malezas. Las canastas fueron depositadas en el suelo y recolectadas a $\operatorname{los} 7,14,21,35$ y 56 días en dependencia de la velocidad de descomposición. Los datos de biomasa seca recolectados de las canastas y su correspondiente contenido de nitrógeno, fósforo, potasio, calcio y magnesio se sometieron a análisis estadístico, resultando el más apropiado el modelo doble exponencial decreciente para la descomposición de la biomasa, donde se reflejan dos coeficientes de descomposición representando el primero a la fracción lábil y el segundo a la fracción recalcitrante. El modelo asintótico se ajustó

\begin{abstract}
Increased understanding of surface residue decomposition and mineralization may improve ground cover management of coffee plantation growing under shade. The first function of ground covers in coffee plantations is to prevent soil erosion, the second function is to improve soil fertility through nutrient cycling, and the third one is to reduce the amount of chemical products to control the most competitive weeds. This experiment of weed biomass decomposition was carried out in rainy season of 2000, with the main goal of determine the rate of decomposition and nutrient ( $\mathrm{N}, \mathrm{P}, \mathrm{K}, \mathrm{Ca}$ and $\mathrm{Mg}$ ) release pattern of leaves and stems for the most common weeds in coffee plantation of the pacific area of Nicaragua. Leaves and stems of the weeds were separated and oven-drying and 30 grams were placed in litterbags of $30 \mathrm{~cm}$ x $30 \mathrm{~cm}$ x $2.5 \mathrm{~cm}$ deep. Litterbags were placed on the bare soil surface in the inter-rows of coffee plants and were collected every $7,14,21,35$ and 56 days or depending of biomass lost. The most appropriate statistic analysis for decomposition was the double exponential decay model were occurred two decomposition rates (labile and recalcitrant), while nutrient release pattern were fitted by the asymptotic model. The $50 \%$ of biomass lost occurred during the first 14 days and more than $50 \%$ of nutrient release occurred during the first 7 days.
\end{abstract}

Keywords: coffee, shade, weeds, litterbags, nutrient cycling 
mejor a la liberación del nitrógeno, fósforo, potasio, calcio y magnesio. La pérdida rápida de la biomasa ocurrió durante los primeros 14 días y luego se dio una descomposición bastante lenta desde los 14 hasta los 56 días que duró el estudio. La mayor liberación de nutrientes ocurrió en los primeros siete días. En futuros estudios de descomposición y liberación de nutrientes de residuos orgánicos se recomienda utilizar los modelos no lineales como el simple exponencial, doble exponencial y asintótico, por su mejor ajuste al comportamiento de los datos en ecosistemas terrestres.

Palabras clave: café, sombra, malezas, canastas de descomposición, reciclaje de nutrientes

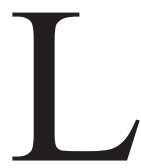

a descomposición y la mineralización de los residuos vegetales, es un aspecto importante en la función de los ecosistemas terrestres. El manejo de residuos vegetales en suelos no disturbados y en cultivos perennes como el café ha sido sujeto de estudio por muchos años debido a los múltiples beneficios en la agricultura sostenible (Unger, 1994). Uno de los principales factores para mantener la fertilidad de los suelos en sistemas agroforestales tropicales es el reciclaje de nutrientes a través de la descomposición de la biomasa. Para medir la descomposición es común usar el método de las canastas metálicas, que consiste en depositar el material vegetal en una canasta de $30 \mathrm{~cm}$ de ancho por $30 \mathrm{~cm}$ de largo y $2.5 \mathrm{~cm}$ de alto, espacio que evita la compactación del material. Aunque el método de las canastas puede subestimar el proceso de la verdadera descomposición que ocurre en el campo, puede reflejar la misma tendencia que el material sin el uso de las mismas.

Aunque existen métodos similares en el diseño y metodología de estudios en canastas de descomposición, existen variaciones en el proceso estadístico utilizado para examinar los datos provenientes de ensayos de descomposición. En el proceso de descomposición los compuestos solubles y relativamente fáciles de degradar como azúcares, almidón y proteínas son fácilmente utilizados por los organismos y microorganismos que degradan la materia orgánica, mientras que otros materiales recalcitrantes como la celulosa, grasas, ceras, taninos y ligninas tienen una tasa de descomposición lenta. Con el tiempo, la proporción relativa del material recalcitrante va progresivamente aumentando y la descomposición absoluta va decreciendo, mientras que la descomposición relativa puede permanecer constante.

El término calidad de un residuo vegetal es comúnmente utilizado cuando se habla de contenido de nutrientes y tasa de descomposición en residuos vegetales (Anderson y Swift, 1983). Residuos vegetales que tienen alto contenido de nutrientes o baja relación $\mathrm{C}: \mathrm{N}$ se descomponen más rápido por lo que son considerados de buena calidad, mientras que otros residuos lignificados que son de difícil descomposición son considerados de baja calidad.
Uso de análisis de varianza para interpretar datos de descomposición. A menudo es frecuente comparar la descomposición de diferentes materiales vegetales y se procede a realizar un análisis estadístico para comparar diferencias entre medias de tratamientos. El método más usado es el análisis de varianza (ANDEVA) con un diseño de Bloques Completos al Azar donde un factor es la calidad del sustrato (A) y el otro factor es el tiempo (B). Los datos de 0 tiempo y $100 \%$ del material inicial no forman parte del grupo de datos por ser iguales para todos los tratamientos.

Supongamos que tenemos los datos de un ensayo en el Centro Experimental del café del pacífico, Jardín Botánico establecido en septiembre del 2000 , donde se pusieron a descomponer solo hojas, solo tallos y hojas más tallos juntos de las principales malezas que crecen en los cafetales por un tiempo de ocho semanas. La cantidad de biomasa depositada en las canastas fue de $30 \mathrm{~g}$ de hojas, $30 \mathrm{~g}$ de tallos y $15 \mathrm{~g}$ de hojas más $15 \mathrm{~g}$ de tallos cuando se mezclaron ambos componentes. En total se distribuyeron en el campo 45 canastas, tres tratamientos, cinco por componente y tres repeticiones. Los $30 \mathrm{~g}$ de la biomasa inicial se tomaron como 100\%. Las muestras fueron recolectadas a $\operatorname{los} 7,14,21,36$ y 56 días después de haber sido distribuidas en el campo, todo material extraño fue retirado de la muestra y ésta fue secada hasta obtener un peso constante a 60 grados Celsius.

Tabla 1. Resultados de peso seco en gramos obtenidos en las anastas después de diferentes períodos de descomposición. Tratamientos (3), recolecciones (5) y bloques (3)

\begin{tabular}{lrrr} 
Días & Hojas & Tallos & Hojas + Tallos \\
\hline 0 & 100.0 & 100.0 & 100.0 \\
7 & 55.2 & 56.2 & 54.0 \\
14 & 45.4 & 45.8 & 44.6 \\
21 & 37.7 & 37.5 & 31.4 \\
35 & 22.9 & 28.6 & 21.4 \\
56 & 18.8 & 24.0 & 17.9 \\
\hline
\end{tabular}

El análisis de varianza contiene los efectos principales del tipo de material como factor A, y tiempo como factor $\mathrm{B}$ y una interacción entre el tipo de material y el tiempo A x B. El análisis de varianza, nos dice que el tipo de material ( $\mathrm{Pr}$ $>0.0170)$ y el tiempo $(\operatorname{Pr}>0.0001)$ son ambos significativos, en cambio la interacción es no significativa $(\operatorname{Pr}>0.7453)$ ver datos adelante. Al encontrar diferencia entre el tipo de material significa que al menos dos de los tres tratamientos difieren en el porcentaje de la biomasa que queda en las canastas y al ser la interacción no significativa la magnitud y dirección de la descomposición es aproximadamente la misma a lo largo de los 56 días de estudio; los valores observados en el campo pueden presenciarse en la figura 1. Cuando la interacción es significativa la dirección de la media del porcentaje de biomasa que queda en la canasta no es la misma a lo largo del tiempo. 
$\mathrm{Al}$ correr el ANDEVA se obtiene el siguiente resultado:

The GLM Procedure

Dependent Variable: DECOMPOSITION

\begin{tabular}{|c|c|c|c|c|c|}
\hline Source & DF & $\begin{array}{l}\text { Sum of } \\
\text { Squares }\end{array}$ & Mean Square & F Value & $\operatorname{Pr}>\mathrm{F}$ \\
\hline$\overline{\text { Model }}$ & 16 & 7777.330222 & 486.083139 & 29.54 & $<.0001$ \\
\hline Error & 28 & 460.799556 & 16.457127 & & \\
\hline Corrected Total & 44 & 8238.129778 & & & \\
\hline $\begin{array}{l}\text { R-Square } \\
0.944065\end{array}$ & & $\begin{array}{ll}\text { Coeff Var } & \text { Root MSE } \\
11.23681 & \end{array}$ & $\begin{array}{l}\text { DECOM Mean } \\
4.056738\end{array}$ & & 36.10222 \\
\hline Source & $\mathrm{DF}$ & Type I SS & Mean Square & F Value & $\operatorname{Pr}>F$ \\
\hline TRAT & 2 & 155.648444 & 77.824222 & 4.73 & 0.0170 \\
\hline TIME & 4 & 7528.576444 & 1882.144111 & 114.37 & $<.0001$ \\
\hline TRAT*TIME & 8 & 82.998222 & 10.374778 & 0.63 & 0.7453 \\
\hline BLOQUE & 2 & 10.107111 & 5.053556 & 0.31 & 0.7380 \\
\hline
\end{tabular}

Según la figura 1, la tasa de descomposición relativa a lo largo del tiempo fue más baja en los tallos y más alta para la mezcla de hojas y tallos de las malezas. El resultado de la descomposición absoluta al final del estudio fue hojas + tallos $>$ hojas $>$ tallos.

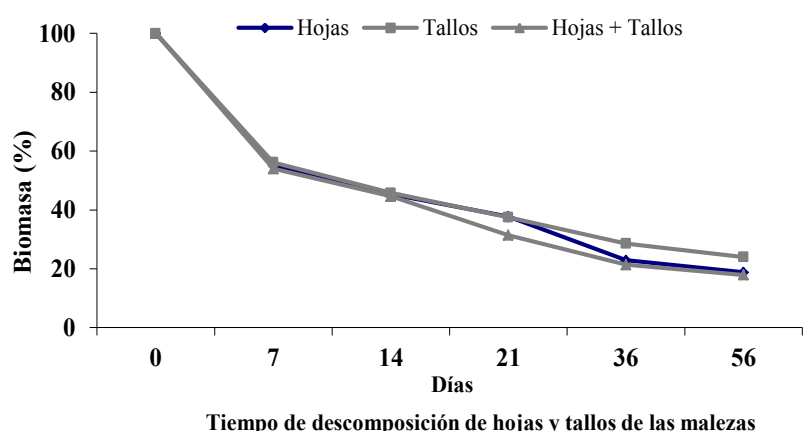

Figura 1. Descomposición de hojas, tallos y hojas + tallos de las principales malezas en el cultivo de café bajo sombra.

Un segundo análisis que podría utilizarse es la ecuación de regresión lineal simple, tomando el tiempo (variable independiente) como X y la tasa de descomposición (variable dependiente) como $\mathrm{Y}$, así mismo se pueden hacer ajustes de curvas para ver si la regresión obedece a una ecuación cuadrática o cúbica tomando en cuenta el mayor valor de r-cuadrado. En ambos casos $\mathrm{Y}$ es el valor de biomasa restante en la canasta, $\beta^{\circ}$ es el intercepto cuando X o el tiempo es cero y $\beta^{1}$ es la pendiente o curva de descomposición. Es importante observar en la figura, la distribución de los datos observados en campo para ver si la curva se comporta como:

$\begin{array}{ll}\text { Línea recta } & Y=\beta_{0}+\beta_{1} X+\varepsilon \\ \text { Curva cuadrática } & Y=\beta_{0}+\beta_{1} X+\beta_{2} X^{2}+\varepsilon \\ \text { Curva cúbica } & Y=\beta_{0}+\beta_{1} X+\beta_{2} X^{2}+\beta_{3} X^{3}+\varepsilon\end{array}$

Estos modelos lineales son menos utilizados para simular descomposición y liberación de nutrientes de residuos orgánicos. El modelo lineal considera que la tasa de la descomposición absoluta es constante mientras que la tasa de descomposición relativa incrementa a través del tiempo. Este supuesto no se puede justificar biológicamente. Cuando algunos residuos orgánicos se descomponen lentamente como los fustes de los árboles es posible encontrar un ajuste lineal pero en la mayoría de los casos solo ocurre durante los primeros años. Al contrario cuando se prueban materiales de fácil descomposición ocurre al inicio una rápida pérdida de la biomasa $\left(\mathrm{k}_{1}\right)$ y posteriormente una pérdida lenta de la biomasa $\left(\mathrm{k}_{2}\right)$ $\mathrm{Al}$ correr el análisis de regresión obtenemos el siguiente resultado:

Procedimiento GLM

Variable dependiente: Biomasa restante en la canasta

\begin{tabular}{|c|c|c|c|c|c|}
\hline Fuente & DF & $\begin{array}{l}\text { Suma de } \\
\text { cuadrados }\end{array}$ & $\begin{array}{l}\text { Cuadrado de } \\
\text { la media }\end{array}$ & F-Valor & $\operatorname{Pr}>F$ \\
\hline Modelo & 1 & 3113.436582 & 3113.436582 & 8.48 & 0.0436 \\
\hline Error & 4 & 1468.971751 & 367.242938 & & \\
\hline Total correcto & 5 & 4582.408333 & & & \\
\hline R-cuadrado & Coef Var & Raíz MSE & \multicolumn{2}{|c|}{ Peso seco Media } & \\
\hline 0.679432 & 42.6964 & 19.16358 & & 44.88333 & \\
\hline & \multicolumn{4}{|c|}{ Cuadrado de } & \multirow[b]{2}{*}{$\operatorname{Pr}>\mathrm{F}$} \\
\hline Fuente & DF & Tipo I SS & la media & F-Valor & \\
\hline DIAS & 1 & 3113.436582 & 3113.436582 & 8.48 & 0.0436 \\
\hline Parámetro & Estimador & $\begin{array}{l}\text { Error } \\
\text { estándar }\end{array}$ & Valor $\mathrm{t}$ & $\operatorname{Pr}>|t|$ & \\
\hline Término in & 71.88132296 & 12.13189875 & 5.92 & 0.0041 & \\
\hline Días - & 1.21795442 & $0.41829989-$ & 2.91 & 0.0436 & \\
\hline
\end{tabular}

Al correr un análisis lineal simple para la descomposición de las hojas y tallos de malezas juntos, según el ANDEVA nos refleja una precisión de $67.9 \%$, un coeficiente bastante bajo para estimar la tasa de descomposición de la biomasa de las malezas. El modelo según el análisis de $\mathrm{SAS}$ sería biomasa $=71.88$ - 1.2179 (días). Según este modelo cuando inicia el experimento se parte de $71.88 \%$ del material original, cuando en realidad es $100 \%$.

Es muy común hacer figuras para conocer la naturaleza de los datos antes de correr el análisis, conocer la distribución normal de los datos y la homogeneidad de las varianzas. Si en la dispersión de los datos se observa una curvatura bastante suave hacia la derecha o hacia la izquierda, es común por los investigadores realizar una transformación logarítmica (LOG de Y (biomasa)), LOG de X (días)) o LOG de ambas Y y X) a los datos para línealizar la curva. En el caso de que los datos presenten definitivamente un comportamiento no lineal o una curvatura 
muy fuerte, lo ideal es correr los datos utilizando modelos no lineales. La regresión debe ser analizada utilizando los datos de tiempo con los datos de hojas, tallos y Hojas + tallos por separado.

En estudios recientes realizados en Kenia con hojarasca de Cassia siamea y Leucaena leucocephala (Nair, 1993) se menciona que la descomposición de estos residuos vegetales presenta un patrón exponencial con una rápida descomposición durante las primeras seis semanas y una descomposición lenta desde la sexta hasta la semana 18. Conociendo este comportamiento es posible sincronizar entre la demanda por nutrientes por parte del cultivo y el suplemento de los nutrientes por parte de los residuos vegetales con su descomposición y mineralización.

Simulando funciones de descomposición. Un tercer análisis para datos de descomposición es utilizar un modelo matemático para estimar constantes que describen la tasa de descomposición de la biomasa durante el tiempo. Estas aproximaciones son de importante valor en la biología de los procesos de descomposición, el cual no se obtienen con el análisis anterior. Estas aproximaciones además de considerar las propiedades matemáticas describen la relación entre los modelos y la biología de la descomposición de la capa orgánica del suelo.

El primer modelo más frecuentemente utilizado es la función simple exponencial ya sea este creciente o decreciente y que fue propuesta por Jenny et al., (1949) y discutido en detalle por Olson (1963). Este modelo considera que la tasa absoluta de descomposición $\mathrm{K}_{1}$ decrece linealmente cuando la cantidad de sustrato declina o la tasa de descomposición relativa permanece constante. Cuando en el análisis estadístico se incluyen datos de descomposición acumulados en porcentajes o ascendentes (figura 2) hasta alcanzar el 100\% de la biomasa descompuesta se recomienda utilizar el siguiente modelo:

A) $\mathrm{Y}=\mathrm{W}_{\mathrm{o}}\left(1-\exp ^{-\mathrm{k} 1 \mathrm{x}}\right)+\varepsilon$

Modelo simple exponencial ascendente

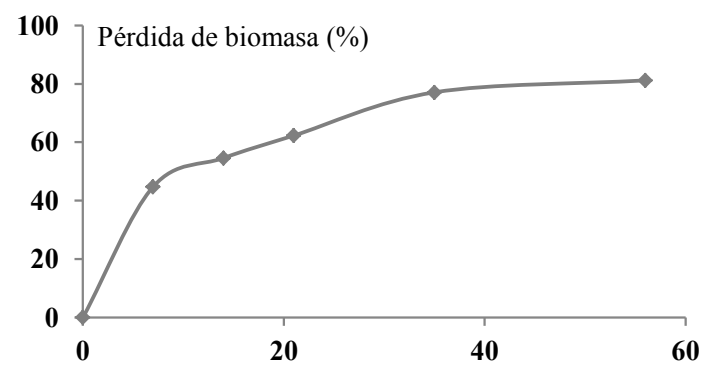

Figura 2. Descomposición acumulada (\%) de la biomasa de hojas de malezas en café.
Corriendo este modelo estadístico simple exponencial ascendente, para la descomposición de hojas de malezas en cafetales (figura 2), se obtiene con un 99\% de precisión el modelo con las constantes $\mathrm{Wo}_{0}=79.0963 \mathrm{y} \mathrm{K}_{1}=0.0929$ con las que se puede predecir la curva de descomposición de las hojas de malezas en cafetales. El tiempo o los días de descomposición es $\mathrm{x}$ en el modelo.

En caso de utilizar en el análisis estadístico, datos descendentes o el valor de la biomasa que queda en la canasta de descomposición se debe utilizar el siguiente modelo:

B) $\mathrm{Y}=\mathrm{W}_{\mathrm{o}} \exp ^{-\mathrm{k} 1 \mathrm{x}}+\varepsilon \quad$ Modelo simple exponencial descendente

Debido a la dinámica de la descomposición, este primer modelo fue modificado a un modelo de descomposición doble exponencial. El modelo doble exponencial asume que la descomposición puede representarse en dos componentes, una fracción lábil de fácil descomposición (A) y otra recalcitrante de difícil descomposición $(1-\mathrm{A})$. La descomposición total es expresada por la suma de las dos tasas de descomposición. En estudios realizador por Aguilar et al., (2001) encontraron que la descomposición de hojas y tallos de las malezas y coberturas nobles en sistemas agroforestales como café se ajustan mejor al modelo doble exponencial.

C) $\mathrm{Y}=\mathrm{W}_{\mathrm{o}} \exp ^{-\mathrm{k} 1 \mathrm{x}}+\left(100-\mathrm{W}_{\mathrm{o}}\right) \exp ^{-\mathrm{k} 2 \mathrm{x}}+\varepsilon \quad$ Modelo doble exponencial

Otro modelo matemático utilizado en los datos de descomposición es el modelo asimptótico (Howard y Howard, 1974). Este modelo, es bastante cercano al modelo simple exponencial y al doble exponencial. El modelo asimptótico es bastante parecido al modelo simple exponencial con constante positiva pero no llega a cero o puede ser como la doble exponencial donde $\mathrm{K}_{2}$ iguala a cero o sea que la fracción recalcitrante es completamente resistente a la descomposición o inmune a la actividad microbiana. Aguilar et al., (2001) encontraron que la liberación del nitrógeno, fósforo, potasio, calcio y magnesio de los residuos orgánicos de las malezas se ajustaron mejor al modelo asimptótico.

D) $\mathrm{Y}=\mathrm{W}_{\mathrm{o}}+\left(100-\mathrm{W}_{\mathrm{o}}\right) \exp ^{-\mathrm{k} 1 \mathrm{x}}+\varepsilon \quad$ Modelo asimptótico

Para los modelos simple y doble exponencial, la tasa de descomposición absoluta tiende a cero a través del tiempo y los modelos matemáticos son casi similares. La tasa de descomposición $\mathrm{K}_{1}$, es constante para el modelo simple exponencial y el valor es casi constante para el modelo doble exponencial $\mathrm{K}_{2}$ a medida que la fracción lábil disminuye. En general los tres modelos son similares y en los tres modelos la descomposición absoluta tiende a cero a medida que el tiempo avanza. 
Tomando en cuenta el modelo simple exponencial, doble exponencial y asimptótico arriba descritos (B, C y D) y utilizando los datos de la tabla 1 para el tiempo y la descomposición de hojas, se procedió a aplicar los tres modelos para observar el mejor ajuste de las curvas. Como se puede ver en la salida de SAS 9.1, el modelo que mejor se ajusta es el que los valores observados sean similares a los valores esperados, que el cuadrado medio sea lo más bajo posible y el coeficiente de determinación $\left(\mathrm{r}^{2}\right)$ sea el más alto.

Análisis de varianza utilizando el Modelo doble exponencial DOUBLE EXPONENTIAL DECAY MODEL The NLIN Procedure

\begin{tabular}{|c|c|c|c|c|c|}
\hline Source & DF & $\begin{array}{l}\text { Sum of } \\
\text { Squares }\end{array}$ & $\begin{array}{l}\text { Mean } \\
\text { Square }\end{array}$ & F Value & $\begin{array}{l}\text { Approx } \\
\operatorname{Pr}>F\end{array}$ \\
\hline Regression & 3 & 17389.2 & 5796.4 & \multirow[t]{4}{*}{956.66} & \multirow[t]{4}{*}{$<.0001$} \\
\hline Residual & 3 & 18.1770 & 6.0590 & & \\
\hline Uncorrected Total & 6 & 17407.3 & & & \\
\hline Corrected Total & 5 & 4340 & & & \\
\hline Parameter & Estimate & $\begin{array}{c}\text { Approx } \\
\text { Std Error }\end{array}$ & \multicolumn{3}{|c|}{$\begin{array}{c}\text { Approximate } 95 \% \text { Confidence } \\
\text { Limits }\end{array}$} \\
\hline W0 & 91.2107 & 8.4830 & & 67.6585 & 114.8 \\
\hline K1 & 0.0437 & 0.00870 & & 0.0195 & 0.0678 \\
\hline
\end{tabular}

Análisis de varianza utilizando el Modelo doble exponencial

DOUBLE EXPONENTIAL DECAY MODEL The NLIN Procedure

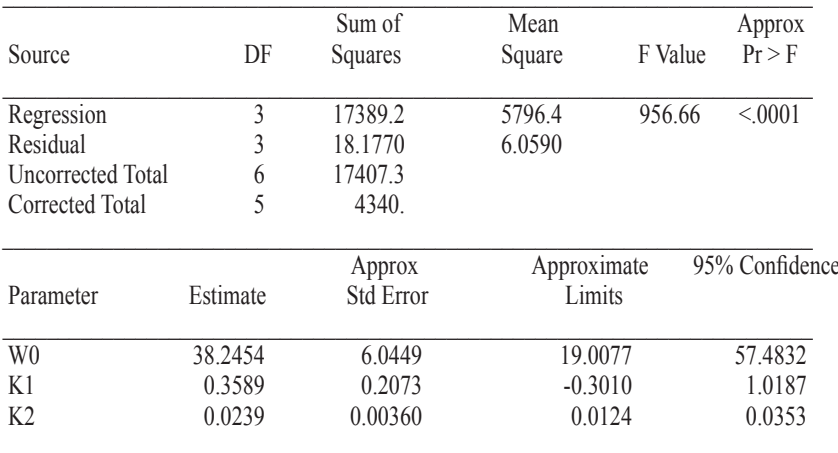

Análisis de varianza utilizando el Modelo asimptótico ASYMPTOTIC MODEL The NLIN Procedure

\begin{tabular}{lrcrrr}
\hline Source & DF & $\begin{array}{c}\text { Sum of } \\
\text { Squares }\end{array}$ & $\begin{array}{c}\text { Mean } \\
\text { Square }\end{array}$ & F Value & $\begin{array}{c}\text { Approx } \\
\text { Pr }>\text { F }\end{array}$ \\
\hline $\begin{array}{l}\text { Regression } \\
\text { Residual }\end{array}$ & 2 & 17312.0 & 8656.0 & 363.03 & $<.0001$ \\
Uncorrected Total & 4 & 95.3736 & 23.8434 & & \\
Corrected Total & 6 & 17407.3 & & & \\
& 5 & 4340.7 & & & \\
\hline & & & & & \\
Parameter & Estimate & Std Error & Approximate & $95 \%$ Confidence \\
& & & & & \\
\hline W0 & 21.0190 & 4.0004 & 9.9123 & 32.1258 & \\
K1 & 0.0931 & 0.0151 & 0.0511 & 0.1351 & \\
& & & & & \\
\hline
\end{tabular}

Con el modelo doble exponencial se obtiene el valor más bajo del cuadrado medio de los residuales (Mean Square Residual) con 6.059, una probabilidad altamente significativa $(\operatorname{Pr}=<0.0001)$ y un valor más alto del coeficiente de determinación $\left(\mathrm{r}^{2}\right)$ con $99 \%$. En segundo lugar la curva de mejor ajuste lo representa el modelo asimptótico con un cuadrado medio de los residuales de 23.8434 y un coeficiente de determinación $\left(\mathrm{r}^{2}\right)$ de $99 \%$ y en tercer lugar el modelo simple exponencial con un cuadrado medio de los residuales de 93.4045 y un mayor coeficiente de determinación $\left(\mathrm{r}^{2}\right)$ con un $97 \%$. Por lo tanto el modelo que mejor se ajusta a la descomposición de las malezas es el doble exponencial.

Utilizando el modelo doble exponencial para la descomposición de los tallos se tiene para $\mathrm{W}_{\mathrm{o}}$ que es el peso de biomasa que todavía queda en la canasta es 49.4806, el valor para la descomposición rápida $\left(\mathrm{K}_{1}\right)$ es 0.2124 y para la descomposición lenta $\left(\mathrm{K}_{2}\right)$ es 0.0142 y para la descomposición de las hojas y los tallos juntos es 48.9065 para $\mathrm{W}_{\mathrm{o}}, 0.2082$ para la descomposición rápida $\left(\mathrm{K}_{1}\right)$ y 0.0211 para la descomposición lenta $\left(\mathrm{K}_{2}\right)$. Los datos esperados o predichos para los tres tratamientos según las ecuaciones dobles exponenciales se presentan en la figura 3 .

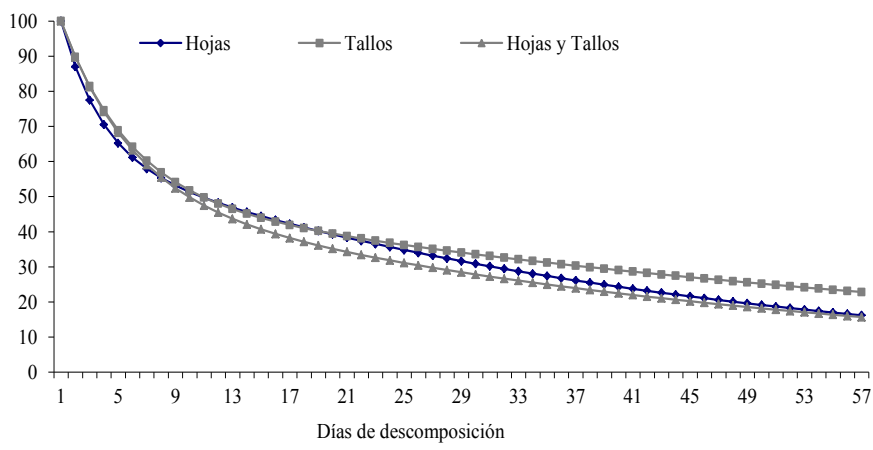

Figura 3. Descomposición de hojas y tallos de malezas durante ocho semanas en un cafetal del Centro Experimental del Café del Pacífico, Masatepe, Masaya, 2002.

De la misma manera se procedió a utilizar los tres modelos matemáticos descritos anteriormente para simular liberación de los nutrientes $(\mathrm{N}, \mathrm{P}, \mathrm{K}, \mathrm{Ca}$ y $\mathrm{Mg})$ de la biomasa de las malezas. Estos valores fueron mejor representados por el modelo asintótico encontrándose en la salida de SAS el valor más bajo del cuadrado medio de los residuales y el coeficiente de determinación $\left(\mathrm{r}^{2}\right)$ más alto. El comportamiento de la liberación de los nutrientes se pueden observar en la figura 4 al utilizar EXCEL sustituyendo los valores del modelo de mejor ajuste.

Los modelos estadísticos antes mencionados se pueden dividir en modelos lineales y modelos no lineales. En los modelos lineales que presenten curvatura hacia la izquierda o hacia la derecha, los datos de biomasa pueden ser transfor- 
mados a logaritmo u otra transformación adecuada y convertirlos en modelos lineales, pero los modelos estrictamente no lineales no pueden convertirse en modelos no lineales a través de transformaciones (Kelman y Lang, 1992 and Draper and Smith, 1966). Observe que estos modelos pueden simular la descomposición cuando el tiempo es cero o sea cuando se tienen un $100 \%$ de la biomasa sin descomponer, de no ser así es posible que el modelo usado no sea el más apropiado como es el caso de modelos con datos transformados.

Los modelos exponenciales son los más apropiados en términos matemáticos y biológicos. Es recomendable hoy en día, el uso de modelos apropiados ya que se disponen de paquetes estadísticos muy poderosos como el SAS 9.1, MINITAB 13, STADISTICA y SSPS y de computadores con gran capacidad para correr los mismos. El uso intensivo de las estadísticas como una herramienta de trabajo es una cosa habitual, mas sin embargo los análisis estadísticos solo proveen una fuente para examinar los datos y pueden ser útiles para interpretar la información recopilada en un determinado estudio. Es requisito conocer las limitaciones y desventajas de un proceso estadístico particular para hacer una interpretación apropiada de los datos.

Se pueden recomendar las canastas de descomposición (litter bags) para hacer estudios de descomposición ya que además de controlar mejor la biomasa que queda en la canasta, la no compactación del material, se simula una des- composición normal permitiendo la entrada de micro y macroorganismos.

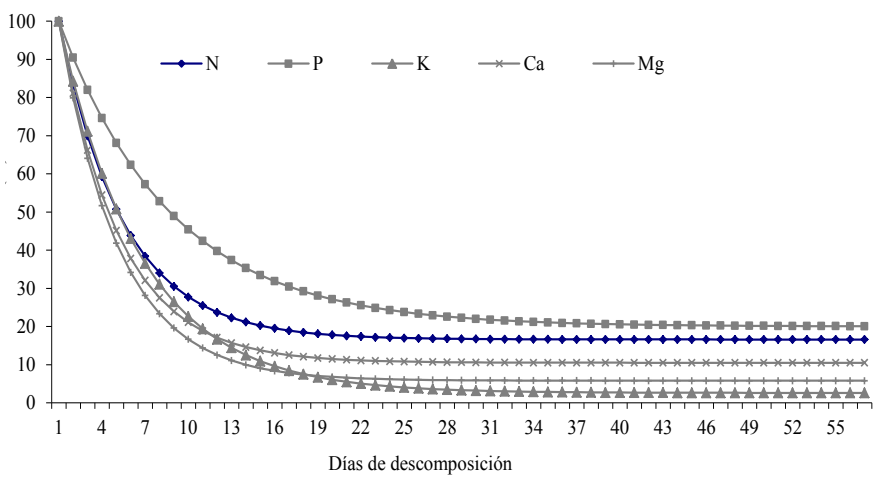

Figura 4. Liberación de nutrientes de hojas y tallos de malezas en café bajo sombra.

\section{CONCLUSIONES}

Cuando están presentes las hojas y tallos de las malezas ocurre una mayor descomposición ya que el mayor contenido de nitrógeno en las hojas mejora la calidad del sustrato. El modelo estadístico que más se ajustó a los datos de descomposición de los residuos de las malezas fue el doble exponencial. El modelo estadístico que más se ajustó a los datos de liberación de los nutrientes fue el asimptótico.

\section{REFERENCIAS BIBLIOGRÁFICAS}

Aguilar, V; C, Staver. 2001. Weed residue decomposition in shaded coffee as affected by residue type, quantity and placement. In Agui lar V. 2001. Selective weed and ground cover management in a coffee plantation with shade trees in Nicaragua. Agraria 269, Swe dish University of Agricultural Sciences. Tesis de doctorado en SLU, Suecia.

Aguilar, V; Staver, C; Milberg, P. 2001. Decomposition and nutrient release dynamics of weeds in shaded coffee. In Aguilar V. 2001. Selective weed and ground cover management in a coffee plantation with shade trees in Nicaragua. Agraria 269, Swedish Univer sity of Agricultural Sciences. Tesis de doctorado en SLU, Suecia.

Anderson, JP; Swift, MJ. 1983. Decomposition in tropical forests. In Sutton S. L., T. C. Whitmore and A. C. Chadwick (eds) Tropical Rain Forest: Ecology and Management. Blackwell, Oxford, UK. p 287-309.

Draper, NR, H, Smith. 1966. Applied regression analysis, John Wiley and Sons, New York USA.

Howard P, JA, Howard, DM. 1974. Microbial decomposition of tree and thrub lraf litter. I. weight loss and chemical composition of decomposing litter. Oikos 25:341-352.

Jenny, H, SP, Gessel, FT, Bingham. 1949. Comparative study of decomposition of organic matter in temperate and tropical regions. Soil Science 68:419-432.

Kelman, R; Lang, GE. 1982. A critique of the analytical methods used in examining decomposition data obtained from litter bags. Ecology. p 1636-1642.

Nair R, PK. 1993. An introduction to Agroforestry. ICRAF, Kluwer Academic Publishers. 499 p.

Olson, JS. 1963. Energy storage and the balance of producers and decomposers in ecological systems. Ecology 44:322-331.

Unger, PW. 1994. Managing agricultural residues. Lewis Publ. Boca raton, Fl: CRC Press. 